\title{
Sarcoidosis of the paranasal sinuses treated with hydroxychloroquine
}

\author{
Sergio Hassid, Georges Choufani, Sven Saussez, Martine Dubois, Isabelle Salmon, \\ Alain Soupart
}

\begin{abstract}
Summary
A case of sarcoidosis of the paranasal sinuses is reported. Biopsies of the sinus mucosa showed typical noncaseating granulomas. Hydroxychloroquine, which is known to be active on the cutaneous form of sarcoidosis, was used here with success and is proposed as an effective alternative to high-dose systemic steroids.
\end{abstract}

Keywords: sarcoidosis; paranasal sinuses; hydroxychloroquine

The aetiology of sarcoidosis, which was first described by Besnier in 1889, remains unknown, although many authors have suggested the influence of genetic and environmental factors. The incidence and the prevalence of systemic sarcoidosis are $2-10$ new cases per 100000 and 40 cases per 100000 , respectively. It occurs in young adults in the $20-40$ year age group $^{12}$ and is more frequently diagnosed in Black, Hispanic, Irish, West Indian and Scandinavian people. ${ }^{34}$

Sarcoidosis is a systemic, granulomatous disease involving mostly mediastinal and peripheral lymph nodes, lungs, liver, spleen, skin, eyes, phalangeal bones and parotid glands. Important laboratory abnormalities are hypercalcaemia, hypercalciuria, hypergammaglobulinaemia, reduced peripheral T-lymphocyte function, and increased levels of angiotensinconverting enzyme (ACE). ${ }^{5}$

The diagnosis of sarcoidosis is established by anatomopathological examination of a tissue sample and the demonstration of a characteristic granuloma without caseation containing giant and epitheloid cells with round or oval nuclei and prominent nucleoli. ${ }^{35}$ Associated clinical manifestations include erythema nodosum, ocular involvement, and arthralgia. Head and neck localisations are rare. In a series reported by $\mathrm{McCaffrey}$ and $\mathrm{McD}$ onald, ${ }^{5}$ only 220 (9\%) out of 2319 patients with sarcoidosis presented involvement of this site. Nasal sarcoidosis represented $2.1 \%$ of all cases while laryngeal sarcoidosis represented $1.2-1.4 \%$, as assessed by Weiss ${ }^{3}$ and Maycock et al. ${ }^{6}$ Gordon estimated the incidence of nasal sarcoidosis to vary from $3 \%$ to $20 \%$ of cases with systemic sarcoidosis, ${ }^{4}$ while McCaffrey and McDonald state that the nasal mucosal lesions of sarcoidosis have not been frequently recognised and the incidence of this manifestation is uncertain. ${ }^{5}$
Nasal sarcoidosis is a chronic granulomatous disease which is commonly associated with other sarcoid manifestations, involvement of the laryngeal mucosa, facial skin, and neck and hilar nodes. ${ }^{7}$ The treatment of upper respiratory tract sarcoidosis has consisted of systemic steroids, and in some cases, topical beclomethasone diproprionate. Chloroquine may suppress some aspects of the disease but induces variable eye effects.

We report a patient with biopsy-proved sinus involvement by sarcoidosis which responded to the administration of hydroxychloroquine, a less aggressive approach than corticosteroid therapy.

\section{Case report}

A 54-year-old black man was referred to our Ear, Nose and Throat department in 1991 for the treatment of a fistula between the oral cavity and the right maxillary sinus secondary to a dental extraction. He had first been evaluated eight years before in another institution for cutaneous lesions (nodules on forearms and head). Pathological analysis demonstrated sarcoidosis involvement of the cutaneous nodules. At this time, the serum ACE level was $36 \mathrm{U} / 1$ (normal value $<25 \mathrm{U} / \mathrm{l}$ ) and bilateral mediastinal lymphadenopathy was recorded. One year later, functional endonasal sinus surgery, combined with a Caldwell-Luc approach, was performed for his chronic sinusitis, without any improvement of his symptoms. There was no evidence of sarcoidosis involvement of sinuses at that time.

On admission to our department, the patient complained of a bilateral purulent nasal discharge which was confirmed by fibre-optic endoscopy. On examination, no lymphadenopathy was found. No other physical abnormality was recorded except for the presence of cutaneous nodules (1-5 mm diameter) on the forehead, nose and the eyelid of the right eye. A sinus computed tomography (CT) scan demonstrated the presence of a pansinusitis (figure 1). Anatomopathological examination of tissue sample obtained by biopsies from the right maxillary sinus showed a mucosa containing noncaseating granulomas. This procedure confirmed the diagnosis of paranasal sinuses sarcoidosis.

Laboratory tests revealed leucopenia (white cell count $3.0 \times 10^{9} / 1,43 \%$ neutrophils, $23 \%$ lymphocytes, $30 \%$ monocytes, $4 \%$ eosinophils); 
Figure 1 Coronal CT scan of the sinuses showing a pansinusitis. One can see the bony erosion of the maxillary sinuses secondary to an old Caldwell-Luc operation

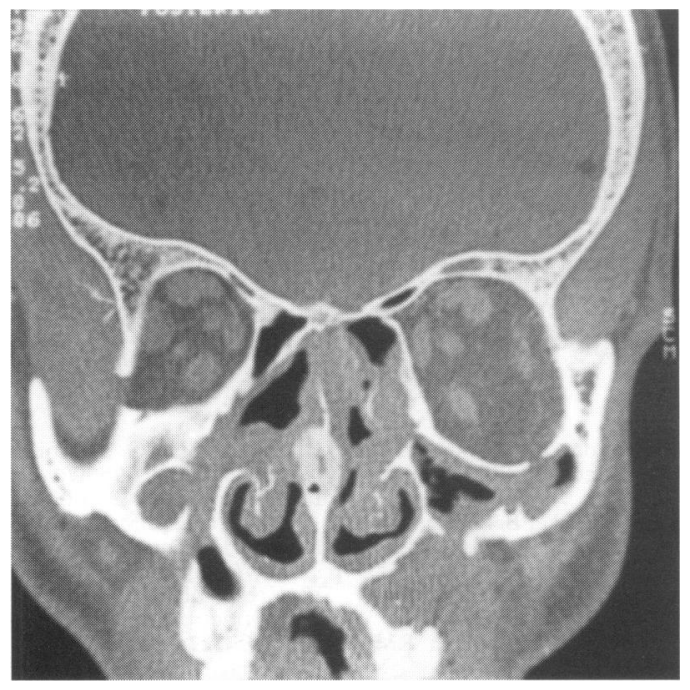

Figure 2 Coronal CT scan of the sinus after treatment

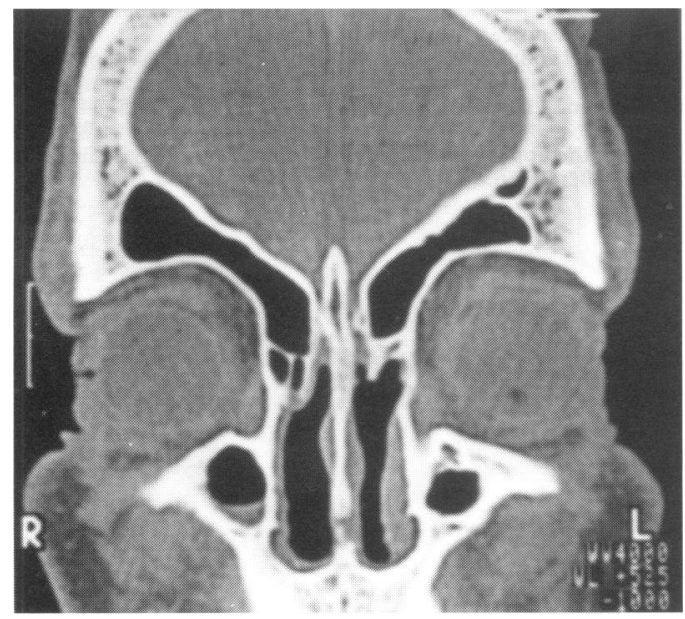

the serum ACE level was $38 \mathrm{U} / 1$ and the level of antineutrophil cytoplasmic antibodies was normal. A tuberculin skin test was negative without previous BCG. Microbiological tests disclosed no mycobacteria, even after long-term cultures. In the absence of bacterial pathogens, no antibiotics were used at any point of treatment. Chest $\mathrm{X}$-ray showed the presence of enlarged mediastinal and hilar lymph nodes, confirmed by chest CT scan. Gallium scintigraphy demonstrated a diffuse tracer uptake in both sides of the pulmonary apexes and in the right hilar region. Lung function tests, including carbon monoxide diffusion capacity, were normal. As pulmonary involvement was not clinically significant, corticosteroid therapy was not prescribed at that time. Hydroxychloroquine, known to be active mainly on the cutaneous forms of sarcoidosis, was then administered at a dose of $200 \mathrm{mg}$ orally bid for one month and then once a day. This treatment was well supported with no ocular complications and led to progressive cure of the fistula and disappearance of the clinical signs of pansinusitis.

Control biopsies performed in the right maxillary sinus were normal. Treatment was discontinued after eight months without adverse effect or recurrence. Chest $\mathrm{X}$-rays and CT scan (figure 2) performed at this time

Learning points
- head and neck involvement in sarcoidosis is
reported in $9 \%$ of cases
sarcoidosis of the paranasal sinuses generally
manifested by recurrent episodes of sinusitis
- hydroxychloroquine could be effective in the
control sinuses involvement of sarcoidosis,
avoiding corticosteroid use

showed persistence of hilar lymph node enlargement. During three years of follow-up the patient did not receive any specific treatment and did not relapse.

In November 1994 he presented with new episodes of recurrent sinusitis. Biopsies performed in the right maxillary sinus demonstrated a recurrence of sarcoidosis in this area. The patient received a second trial of hydroxychloroquine ( $200 \mathrm{mg}$ orally once a day) for five months with a similar favourable response (negative biopsies after five months). Hydroxychloroquine was discontinued and he is still (June 1997) free of symptoms.

\section{Discussion}

In the large series reported by $\mathrm{McCaffrey}$ and McDonald, ${ }^{58}$ head and neck involvement were demonstrated in $9 \%$ of cases of sarcoidosis. When present, the affected sites included lacrimal glands $(40 \%)$, skin $(26 \%)$, nose $(13 \%)$, central and peripheral nervous system $(6 \%)$, larynx $(6 \%)$, salivary glands $(4 \%)$, cervical lymph nodes $(4 \%)$, and the middle ear (1\%). According to these authors, nose and paranasal sinus involvement occurs in about $1 \%$ of patients. Other authors have described abnormal sinus X-rays with thickening of the mucosa attributed to sarcoidosis but this was not always proved by biopsy. ${ }^{8-12}$ Recently, Krespi et $a l^{13}$ have proposed a staging system for sarcoidosis of the sinonasal tract. According to these authors, our case is a stage III because of multiple sinus involvement and should be treated by systemic corticosteroids.

Differential diagnosis of the granulomatous diseases occurring in the upper respiratory tract includes tuberculosis, leprosy, syphilis, Wegener's granulomatosis and sarcoidosis. ${ }^{11}$

All these aetiologies other than sarcoidosis were excluded in this reported case. When the diagnosis of sarcoidosis is suspected, based on the patient's history, biopsy should be performed prior to surgery. We think that, in this case, the functional endonasal sinus surgery performed in the other hospital was not helpful.

Corticosteroids are the most effective drugs in the treatment of sarcoidosis. Usually, 20-40 $\mathrm{mg}$ of prednisolone are given daily for three to six months. Other drugs such as chloroquine or hydroxychloroquine, azathioprine, and methotrexate, have been used, with varying success. ${ }^{11415}$

To our knowledge, this is the first reported case of sarcoidosis of the paranasal sinus to be successfully treated with hydroxychloroquine. 
This treatment led to the cure of the sinus involvement, but not of the mediastinal lymph node involvement or pulmonary sarcoidosis. As the patient only complained of sinusitis, this treatment was thus appropriate. The response to the second trial of hydroxychloroquine strengthens the evidence of its efficacy in this indication. Following this observation, we suggest that upper respiratory tract mucosal sarcoidosis can be successfully treated with

1 James DG, Barter S, Jash D, Mc Kinnon DM, Carstairs LS. Sarcoidosis of the upper respiratory tract (SURT). F Laryngol Otol 1972;96:711-8.

2 Hillerdal G, Nou E, Osterman K, Schmekel B, Sarcoidosis: epidemiology and prognosis. Am Rev Resp Dis 1984;130:2932 .

3 Weiss JA. Sarcoidosis; an assessment of the value of blind biopsy. Laryngoscope 1960;70:1351-98.

4 Gordon WW, Cohn AM, Greenberg SD, Komorn RM Gordon WW, Cohn AM, Greenberg SD, Kom
Nasal sarcoidosis. Arch Otolaryngol 1976;102:11-4.

5 McCaffrey TV, McDonald TJ. Sarcoidosis of the nose and McCaffrey TV, McDonald TJ. Sarcoidosis of the
paranasal sinuses. Laryngoscope 1983;93:1281-4.

Maycock RL, Bertrand P, Morrison CE, Scott JH. Manifestations of sarcoidosis. Am $\mathcal{F}$ Med 1963;35:67.

McKelvie P, Gresson C, Pokhrel RP, Jackson P. Sarcoidosis of the upper air passages. Br $\mathcal{F}$ Dis Chest 1968;62:200.

8 Bordley JE, Proctor DF. Destructive lesion in the paranasal sinuses associated with Boeck's 'Sarcoid'. Arch Otolaryngol 1992;36:740-4.

9 Lindsay JR, Perlman H. Sarcoidosis of the upper respiratory tract. Ann Otol Rhinol Laryngol 1951;60:549-66. hydroxychloroquine which was until now considered to be active mainly on the cutan eous forms of sarcoidosis. Hydroxychloroquine is extensively used for its immunosuppressive effects in various autoimmune disorders. Ocular toxicity (keratopathy, retinopathy) is unlikely to occur at the 'low' daily dosage used here (200-400 mg/day), even on long-term therapy (more than six months) when regular ocular testing is performed. ${ }^{16}{ }^{17}$

10 Livingstone G. Sarcoidosis of the maxillary antrum. $\mathcal{F}$ Laryngol Otol 1956;70:426-9.

11 Maillard AAJ, Goepfert H. Nasal and paranasal sarcoidosis. Arch Otolaryngol 1978;104:197-201.

12 Tay HL, Vaughan-Jones R, Qureshi SS. Ethmoidal sarcoido-

sis. $\mathcal{F}$ Laryngol Otol 1994;108:682-4.
13 Krespi YP, Kuriloff DB, Aner M. Sarcoidosis of the sinonasal tract: a new staging system. Otolaryngol Head Neck Surg 1995;112:221-7.

14 Morse SI, Cohn ZA, Hirsch JG, Schaedler RW. The treatment of sarcoidosis with chloroquine. Am f Med 1961; 33: 779-84.

15 Fenton DA, Shaw M, Black MM. Invasive nasal sarcoidosis treated with methotrexate. Clin Exp Dermatol 1985;10:279 83.

16 Bernstein HN. Ophthalmologic considerations and testing in patients receiving long-term antimalarial therapy. $A m \mathcal{F}$ Med 1983;74:25-34.

17 Maksymowych W, Russel AS. Antimalarials in rheumathology: efficacy and safety. Semin Arthritis Rheum 1987;16:206-21. 